\title{
Fortschritt, Diskontinuität und Einheit der Wissenschaften
}

\author{
Eine Untersuchung zum Problem wissenschaftlicher Polyperspektivität
}

Thomas Fuchs

\section{SUMMARY}

The development of modern science has been accompanied and essentially motivated by the ideal of a steady and cumulative progress in the knowledge and grasp of nature. However, the crisis of the idea of progress during this century has been reflected in new concepts of the history of science as a discontinuous process of successive systems of thinking, or paradigms. A comparison of T.S. Kuhn's and L. Fleck's models of the history of science, and the example of vitalism vs. mechanistics in the history of physiology (Harvey, Descartes, $v$. Haller) raise the question of whether this "monolinear" course is not an idealization of the factual "polylinear" development of the history of science. As an alternative, the author outlines the possibility of a "complementary evolution" of interpretations of reality which would contribute to a "polyperspective" science.

\section{ZUSAMMENFASSUNG}

Die Entwicklung der abendländisch-neuzeitlichen Wissenschaften ist begleitet und wesentlich motiviert vom Ideal eines kumulativ-stetigen, epochenübergreifenden Erkenntnisfortschritts, einer immer vollständigeren Durchdringung und Erfassung der Natur. Die Krise des Fortschrittsgedankens in unserem Jahrhundert hat sich demgegenüber in neueren Konzeptionen der Wissenschaftsgeschichte als eines diskontinuierlichen Prozesses aufeinanderfolgender Denksysteme oder Paradigmen niedergeschlagen. Ausgehend von einem Vergleich der wissenschaftsgeschichtlichen Modelle von T.S. Kuhn und L. Fleck und gestützt auf das Fallbeispiel von Vitalismus und Mechanizismus in der Physiologiegeschichte (Harvey, Descartes, v. Haller) wird die Frage aufgeworfen, ob dieser nach wie vor "monolinear» konzipierte Verlauf nicht gleichfalls eine Idealisierung der tatsächlichen, "polylinear» verflochtenen Entwicklung der Wissen- 
schaftsgeschichte darstellt. Als Alternative wird die Möglichkeit einer «komplementären Evolution» aspekthafter Wirklichkeitsdeutungen im diachronen Verlauf entworfen, die schliesslich zu einer "polyperspektivischen» Wissenschaft beitragen könnten.

Ist Wissenschaft ohne Fortschritt denkbar? - Diese Frage zu verneinen, käme wohl einer unzulässigen Verkürzung des Wissenschaftsbegriffs gleich. Die grossen wissenschaftlichen Systeme etwa der Antike, der präkolumbianischen oder ostasiatischen Kulturen waren zweifellos hochentwickelt und blieben doch im wesentlichen statische Gefüge, in denen einmal gewonnene Erkenntnisse vertieft und ergänzt, aber nicht grundlegend überholt wurden; Ziel war das systematische Erfassen des Bekannten und alltäglich Vertrauten, nicht das Vordringen ins Unbekannte. - Anders die abendländisch-neuzeitliche Form der Wissensentwicklung: offensichtlich verdankt sie ihre innere Dynamik wie ihren gesellschaftlichen Erfolg wesentlich der Idee eines stetigen Erkenntnisfortschritts, den daraus resultierenden technischen Errungenschaften und nicht zuletzt den an sie geknüpften sozialutopischen Hoffnungen und Erwartungen.

Mit dieser Sonderentwicklung der europäischen Wissenschaft geht allerdings auch eine Beschränkung des ihr je eigenen Blickfeldes einher: ein Nebeneinander konkurrierender Sichtweisen, eine Koexistenz gegensätzlicher Erklärungsmodelle ist dieser Wissenschaft fremd. Auf der Suche nach objektiver bzw. intersubjektiv verbindlicher Wahrheit trachtet sie danach, Widersprüche zu eliminieren oder aber durch synthetisierende Theorien auf höherer Ebene zu überwinden. Dieses restriktive Verhältnis zu möglicher Divergenz, also der Ausschluss simultaner Vielfalt in Theorie und Praxis der Naturwissenschaften liegt letztlich in der Idee des einsinnig-linearen Fortschritts begründet, der durch auftretende Widersprüche in Frage gestellt würde. So kommt es zu dem Phänomen, dass die Naturwissenschaften gleichsam als monolithischer Block im sonst pluralistischen Gefüge der modernen Gesellschaften stehen und nicht zuletzt durch ihre Geschlossenheit eine dominierende Stellung darin einnehmen.

Nun haben jedoch die sozialen und ökologischen Auswirkungen der wissenschaftlich-technischen Innovationen in den letzten Jahrzehnten mehr und mehr die Schattenseiten eines einsinnigen Fortschritts zutage treten lassen. Die daran sich entzündende Kritik führte zur Suche nach Alternativen auch innerhalb der Wissenschaften selbst: nach möglichen, aber im Zuge ihrer monolinearen Entwicklung unentfaltet gebliebenen und in Ver- 
gessenheit geratenen Sichtweisen. Mit anderen Worten: gerade die ausserwissenschaftlichen Fortschrittskosten lenkten die Aufmerksamkeit auch auf die Verluste und Folgelasten, mit denen der innerwissenschaftliche Fortschritt immer einhergegangen war ${ }^{1}$. Eine Kritik der Fortschrittskonzeption muss aber notwendig die Frage aufwerfen, wie der Widerstreit zwischen verschiedenen Sichtweisen und Erklärungsmodellen in Zukunft zu bewerten sei und wie die Gesellschaft mit ihm verfahren soll. - In dieser Situation fällt der Wissenschaftsgeschichte eine bedeutsame Aufgabe zu: nämlich das Verhältnis von Wissenschaft und Widerspruch im bisherigen Geschichtsverlauf zu untersuchen und dabei Erfahrungen über inkongruente Sichtweisen, ihre Konkurrenz und Interaktion zu gewinnen.

Die folgende Studie will dazu in zweierlei Weise beitragen: zum einen durch eine Gegenüberstellung von Thomas S.Kuhns und Ludvik Flecks Konzeptionen der Wissenschaftsgeschichte als eines diskontinuierlichen, von widerstreitenden Paradigmen bzw. Denkstilen geprägten Prozesses; zum anderen durch das Fallbeispiel der Auseinandersetzung von Vitalismus und Mechanismus im Gefolge von William Harveys Entdeckung des Blutkreislaufs. Dabei wird die Frage im Vordergrund stehen, ob sich die Wissenschaft tatsächlich in der monolinearen Form entwickelt hat, wie es dem Betrachter zunächst erscheint, und wie ihr die Theorie immer noch vorschreibt. Abschliessend werden einige mögliche Konseqenzen für den Wissenschafts- und Fortschrittsbegriff formuliert. - Zunächst soll jedoch in einem Rückblick die Idee des linearen Fortschritts beleuchtet werden, von der die bisherigen Konzeptionen der Wissenschaftsgeschichte ihren Ausgang nehmen.

\section{Der Fortschrittsgedanke}

Fortschritt als generationenübergreifender, im Prinzip unbegrenzter Kumulations- und Optimierungsprozess - dieser Gedanke ist ein vergleichsweise neues, spezifisches Produkt der abendländischen Kulturgeschichte. Der bis dahin gleichsam naturwüchsige Fortschritt der Zivilisation wird in der Neuzeit zum ersten Mal als solcher nicht nur bewusst, sondern auch intendiert und zum Programm erhoben. Dieses Programm impliziert, etwa in der Formulierung seines ersten Propagandisten Francis Bacon (Novum Organon 1620), vornehmlich die wissenschaftliche Erkenntnis der Natur einerseits und ihre daraus resultierende technische Beherrschung andererseits. 
Die gezielte Planung und Beschleunigung dieses Fortschritts bedeutet zugleich eine radikale Abwendung von der bis dahin gültigen Konzeption des Wissens. Ihr Bezugspunkt lag in der Vergangenheit: menschenmögliche Erkenntnis hatten die antiken Denker bereits geleistet; schlechthin gültiges Wissen aber war im unüberschreitbaren Gehalt der christlichen Offenbarung tradiert. - Anders der naturwissenschaftliche Erkenntnisprozess: sein Bezugspunkt liegt in der Zukunft, und sich ihm anzunähern gelingt nicht durch Treue zur Überlieferung, sondern gerade durch Misstrauen gegenüber dem Tradierten, durch die Suche nach dem Neuen und Unbekannten: «All das, was man weiss, ist fast nichts im Vergleich zu dem, was noch zu wissen übrig ist», schreibt Descartes im «Discours de la Méthode.» ${ }^{2}$ In diesem nach vorne offenen Prozess ist die Vergangenheit nicht mehr unerreichbares Ideal, sondern eine Geschichte überwundenen Irrtums; in Bacons Formulierung: «Wie viele Irrtümer man in der Vergangenheit auffindet, so viele Hoffnungsgründe erhält man für die Zukunft.» ${ }^{3}$

Diese Wendung hatte sich jedoch schon vorbereitet, nämlich durch eine innere Erosion der bisherigen Wissensform. Differenzen über die Auslegung des Tradierten und Widersprüche zwischen überlieferten Lehren waren im ausgehenden Mittelalter immer schärfer hervorgetreten; dies spiegelte sich etwa in der spätscholastischen Lehre von den «zwei Wahrheiten» der Vernunft und des Glaubens wider (Wilhelm v. Occam, um 1300). Der allmähliche Zerfall der zentralen kirchlichen Autorität, gipfelnd im päpstlichen Schisma Rom-Avignon um 1400, begünstigte das Aufkommen immer neuer Häresien und mündete schliesslich in die Glaubensspaltung und -kriege des 16. und 17. Jahrhunderts. Dieser Verlust weltanschaulicher Einheit stellt ein entscheidendes Trauma der abendländischen Kultur dar, mit einer nachhaltigen Wirkung nicht zuletzt auf die Naturwissenschaften, deren Wiedergeburt, wie Bacon selbst hoffnungsvoll feststellt ${ }^{4}$, eben in die Epoche der Religionsstreitigkeiten fällt. Die neue Wissenschaft erhält nämlich ihren Impetus wesentlich aus dem Versuch einer Überwindung der Spaltung : durch die Abgrenzung eines neuen, autonomen Bereichs von Wissen und Erkenntnis, dessen Verbindlichkeit nun in einer zukünftigen Wahrheit begründet ist, der sich die Wissenschaft in einem kollektiven Prozess asymptotisch annähert.

Bereits Bacon beklagt die bisherige «Uneinigkeit unter den Philosophen», die «Vielfalt der Schulen», die «Gegensätzlichkeit der Dogmen» und stellt ihnen den «verus consensus» der Wissenschaftler gegenüber ${ }^{5}$. Noch deutlicher wird der Anspruch der neuen Wissenschaften von Descartes 
formuliert, der wohl nicht zufällig gerade als Teilnehmer am 30jährigen Krieg die Grundlagen seines Systems entwickelte ${ }^{6}$; er bezeichnet es als die

«... Frucht meiner neuen Naturlehre, dass die darin enthaltenen Wahrheiten sehr klar und sehr gewiss sind und daher jeden Grund zu Streitigkeiten beseitigen, so dass man dadurch zu Milde und Eintracht geneigt wird, während demgegenüber die Schulstreitigkeiten, indem sie nach und nach ihre Jünger immer streitsüchtiger und hartnäckiger machen, vielleicht die erste Ursache der Häresien und Auseinandersetzungen sind, die gegenwärtig die Welt verheeren.»?

Ihre integrierende Kraft verdankt die Wissenschaft wesentlich dem Fortschrittsversprechen:

«Die vorzüglichste Frucht dieser Prinzipien aber ist die, dass man durch ihr Studium eine grosse Zahl von Wahrheiten ... noch entdecken wird, und indem man so nach und nach von ihnen zu anderen fortschreitet,... mit der Zeit zu der höchsten Stufe der Weisheit wird gelangen können.» ${ }^{8}$

Diese wird dann in einer mathematisch-deduktiven Einheitswissenschaft bestehen, welche alle Bereiche der erfahrbaren Wirklichkeit überspannt und einander kommensurabel macht - eine Vorstellung, die etwa in Gestalt einer möglichen «Weltformel» bis ins 20. Jahrhundert weiterwirkt.

Die neuzeitliche Wissenschaft erhebt also bereits in ihren Anfängen den Anspruch, durch Herauslösung der Naturerkenntnis aus dem traditionellen Glaubens- und Lehrgebäude einen neuen «consensus omnium» herzustellen; durch fortschreitende und in sich widerspruchsfreie Naturerkenntnis soll das im Glauben gespaltene Abendland seine Einheit wiederfinden. Das Fortschrittsprogramm wendet sich gegen die brüchig gewordene Tradition geoffenbarten und fixierten Wissens - und setzt sie doch fort in der Anerkennung der einen Wahrheit und im Verbot von Häresien, d. h. im Ausschluss konkurrierender Sichtweisen. Diese werden zu den überwundenen Irrtümern, die die Wissenschaft in ihrem Fortgang im Dunkel der Vergangenheit zurücklässt. Seit der Neuzeit hat die Naturwissenschaft das Privileg, ihre eigene Geschichte zu vergessen, um nur die positiv brauchbaren Resultate zu bewahren und sich auf jedem erreichten Stand für geschichtslos, nämlich für objektiv und absolut zu halten. Die Fortschrittsidee ist also paradoxerweise verknüpft mit einem ahistorischen Selbstverständnis der Wissenschaften und dies wiederum entspricht nur der Geschichtslosigkeit der Offenbarungswahrheit oder der ehemals für zeitlos gehaltenen Gültigkeit antiken Wissens.

Die dynamische Entfaltung der naturwissenschaftlichen Fortschrittsidee muss hier nicht mehr im einzelnen verfolgt werden. Ihre Macht und Faszination wird gerade daran deutlich, dass sie vorübergehend auch auf ausserwis- 
senschaftliche Bereiche übergreifen und sich in der Aufklärung zu einem allgemeinen Geschichtsoptimismus erweitern konnte. Hand in Hand mit dem linearen wissenschaftlichen Fortschritt sollten sich auch Kultur, Gesellschaft und Sittlichkeit in einem «unendlichen Progress» (Kant) vervollkommnen, sollte die «Erziehung des Menschengeschlechts» (Lessing), die «Progression der ganzen Schöpfung» (Herder) voranschreiten ${ }^{9}$. Bereits im Verlauf der französischen Revolution wird jedoch das bürgerliche Ideal eines widerspruchsfreien gesellschaftlichen Fortschritts zunichte. Hegel rettet den Geschichtsoptimismus nur durch Geschichtsdialektik; und Marx sieht die Grunddynamik der Geschichte nun gerade im Missverhältnis zwischen dem linearen (nicht-dialektischen) Fortschritt der wissenschaftlich-technischen Produktivkräfte einerseits und der in sich widerspruchsvollen, durch Revolutionen vorangetriebenen Entwicklung der Gesellschaft andererseits. Erst nach dem «Sprung ins Reich der Freiheit», in der kommunistischen Utopie, werden wissenschaftlich-technischer und gesellschaftlicher Fortschritt Hand in Hand gehen.

Nach den gesellschaftlichen und menschlichen Katastrophen unseres Jahrhunderts ist Fortschritt in seiner universellen Bedeutung zweifelhaft, wenn nicht zunichte geworden. Utopisches Denken vermag sich allenfalls noch in radikaler Entgegensetzung zum bisherigen Geschichtsverlauf zu behaupten. Ungebrochen bleibt der Impetus der Fortschrittsidee nurmehr in ihrem ursprünglichen, wissenschaftlich-technologischen Kernbereich. Und doch sieht sie sich auch hier zunehmender Kritik ausgesetzt, und zwar aus zweierlei Richtungen, die den beiden Komponenten des Baconschen Fortschrittsprogramms entsprechen. Auf der Seite der Naturbeherrschung hat der Übergang vom linearen zum exponentiellen Fortschritt mit seinen Folgelasten die Frage der gesellschaftlichen Wünschbarkeit dieses Fortschritts aufgeworfen. Auf der Seite der Naturerkenntnis aber ist - nicht zuletzt im Gefolge der unvorhergesehenen Krise und Revolutionierung der theoretischen Physik seit Beginn des Jahrhunderts - auch die prinzipielle Möglichkeit stetigen wissenschaftlichen Fortschritts zweifelhaft geworden. Mit den Namen von Kuhn, Lakatos oder Feyerabend verbindet sich eine grundsätzlich neue Konzeption der Wissenschaftsentwicklung als diskontinuierlicher Aufeinanderfolge zeitgebundener Wissenssysteme, von denen sich prinzipiell keines mehr als «objektiver» oder «wahrer» gegenüber den anderen auszeichnen lässt. Auch hierin zeigt sich die neuere Wissenschaftstheorie beeinflusst von der theoretischen Physik, die schon vorher zur 
Aufgabe einer objektiv gegebenen Realität und zur Einbeziehung des Beobachterstandpunkts genötigt war.

Die Krise der Physik als der paradigmatischen Naturwissenschaft hat also in unserem Jahrhundert das abendländische Trauma des verlorenen Konsenses wieder aufbrechen lassen, der im stetigen, generationenübergreifenden Erkenntnisfortschritt gefunden schien. Damit aber stellt sich erneut die Frage von Wahrheit und Widerspruch, von Alleinherrschaft einer oder Vielfalt verschiedener Sichtweisen; Feyerabends «Against Method» ${ }^{10}$ steht als ein Symptom dieser Krise wie ein Kontrapunkt zu Descartes' «Discours de la Méthode». - Wir wollen uns nun den neueren Konzeptionen der Wissenschaftsgeschichte mit der Frage zuwenden, welche Rolle sie dem Widerspruch zwischen verschiedenen Erklärungssystemen zuweisen.

\section{Diskontinuität und Zusammenhang in der Wissenschaftsentwicklung - Thomas S. Kuhn vs. Ludvik Fleck}

\section{a) T.S. Kuhn: Wissenschaftsgeschichte als Paradigmenabfolge ${ }^{11}$}

Mit T.S. Kuhns (Wieder-)Entdeckung der Diskontinuität der Wissenschaftsgeschichte zerfällt das historische Kollektivunternehmen Wissenschaft : an die Stelle des einheitlichen Fortschrittsprogramms tritt die disparate Abfolge einzelner Forschungsprogramme. Erkenntnisfortschritt ist nur möglich innerhalb eines konstanten kognitiven und begrifflichen Rahmens; der Wechsel des Rahmens selbst, also die Paradigmenrevolution, kann nicht mehr als Fortschritt in der Erfassung der Wirklichkeit gelten - die Inkommensurabilität der Paradigmen erlaubt keinen solchen Vergleich.

Trotz dieser diachronen Variabilität des Bezugsrahmens sieht Kuhn nach wie vor in der Konzentration auf ein Paradigma und dem Ausschluss konkurrierender Sichtweisen die entscheidende Voraussetzung für die Entstehung von Wissenschaft. Die Koexistenz gegensätzlicher «Schulen» auf einem Gebiet gehört der "prähistorischen» Phase einer Wissenschaft an ${ }^{12}$; ihre unvereinbaren Ergebnisse begründen «keine gemeinsame Ansicht über die Natur», keinen verbindlichen Forschungshorizont und daher «keine rechte Wissenschaft ${ }^{1{ }^{3}}$ ». Denn in dieser, in der «normalen Wissenschaft», gibt es «gewöhnlich keinen Wettstreit zwischen verschiedenen Ansätzen». ${ }^{14}$

Nur in Phasen des Paradigmenumsturzes fällt eine Wissenschaft vorübergehend in das anarchische Frühstadium konkurrierender Sichtweisen zurück. Dann wird es zu ihrer «Hauptaufgabe ..., erneut alle Regelsysteme bis 
auf eines auszuschalten» ${ }^{15}$ - nämlich um der Effektivität konsensorientierter Forschung willen. Allerdings kann nur ein Akt des «Glaubens» die Widersprüche überwinden: die Annahme eines neuen Paradigmas, von dem die Mehrzahl der Forscher die - unbeweisbare - Überzeugung gewinnt, es sei zur Lösung der Krise, zur Eröffnung eines neuen Forschungshorizonts geeignet ${ }^{16}$. Auch die Wissenschaft entgeht also dem Glaubensstreit nicht: die Alleinherrschaft eines Paradigmas besteht nur auf Zeit, und die ausgeschlossenen Widersprüche brechen periodisch immer wieder auf.

Doch das Fehlen einer rationalen Legitimation des jeweils zur Herrschaft gelangten Paradigmas bleibt dem einzelnen Wissenschaftler später in der Regel verborgen. Er ist befangen zum einen in der Illusion der «objektiven Wahrheit» als der «Quelle für die Überzeugung des Wissenschaftlers ..., dass unvereinbare Regeln für die Ausübung einer Wissenschaft nicht koexistieren können» ${ }^{17}$; zum anderen in der Illusion des «Fortschritts», erzeugt durch eine kollektive Manipulation der Geschichte des eigenen Fachgebiets jeweils im Anschluss an einen Paradigmenumsturz: «... daraus ergibt sich eine manchmal drastische Verzerrung in der Auffassung des Wissenschaftlers von der Vergangenheit seiner Disziplin ... als führte sie in gerader Linie zum gegenwärtigen Stand ${ }^{18}{ }$. - Erst der übergeordnete Blick des Wissenschaftshistorikers durchschaut diese Illusionen. Er erweist Wahrheit als zeitgebunden, Fortschritt als blossen Fortgang von einem Standpunkt zum nächsten, und erkennt doch zugleich die Notwendigkeit dieser Denkbeschränkungen im Interesse einer effektiven Wissenschaftsausübung.

Hier gewinnt Kuhns Konzeption (wie er auch selbst bemerkt ${ }^{19}$ ) durchaus normativen Charakter: Wissenschaft («science») ist per definitionem monoparadigmatische Wissenschaft und muss es bleiben, wenn sie ihre Einheit bewahren soll. Auch bei Kuhn findet somit der Anspruch europäisch-neuzeitlicher Wissenschaft noch einmal eine Fortsetzung; seine Konzeption gleicht einem säkularisierten Monotheismus, dessen Inhalte nun zwar austauschbar geworden sind, an dessen Einheitsprinzip aber formal noch festgehalten wird: an die Stelle der einen Wahrheit ist der historisch relativierte Konsens getreten.

Nun könnte gerade das Schillern des Kuhnschen Textes zwischen normativer und deskriptiver Darstellung den Verdacht nahelegen, auch seine (immer noch monolineare) Konzeption gebe die Wissenschaftsgeschichte in idealisierter Form wieder: Möglicherweise besteht auch hier die Diskrepanz zwischen Anspruch und Wirklichkeit der Wissenschaftsentwicklung weiter fort. Verläuft diese Entwicklung tatsächlich in der geordneten Weise voll- 
ständiger Ablösung eines Paradigmas durch ein neues? - Bevor wir diese Frage an einem historischen Beispiel prüfen, wollen wir noch einen Blick auf die wissenschaftshistorische Konzeption L. Flecks richten, die viele Gedanken Kuhns vorwegnimmt, und zwar weniger systematisiert, dafür aber flexibler und in manchem der historischen Realität adäquater erscheint.

\section{b) Ludvik Flecks «Vergleichende Denkstilforschung» ${ }^{20}$}

Unter «Denkstil» versteht L.Fleck eine dem jeweiligen historisch-sozial definierten Kollektiv eigene, gestaltbildende Wahrnehmungsweise und ein damit verbundenes Begriffssystem, dessen Bestandteile nicht ohne Sinnveränderung in einen anderen Denkstil transferiert werden können. Gegenüber dem Begriff des «Paradigmas» - nach Kuhn v. a. eine als fixierte Theorie oder tradierter Kanon objektiv vorliegende «Problemlösungsanleitung» («puzzle solving») - bezeichnet der «Denkstil» noch deutlicher eine subjektive geistige Bereitschaft, eine Weltsicht oder Sichtweise; er reicht damit auch über den streng naturwissenschaftlich orientierten Paradigmenbegriff hinaus.

Im Vorgriff auf Kuhns Paradigmenrevolution spricht auch Fleck bereits von Epochen der «Denkstilumwandlung», von «Mutationen» der Denkgeschichte und dem dabei auftretenden «Streit der Gesichtsfelder» ${ }^{21}$. Während jedoch Paradigmen weitgehend stabile Einheiten darstellen, betont Fleck die evolutionäre Wandelbarkeit der Denkstile, die z. B. durch Entdekkungen fortwährend verändert werden: «Wenn es eine völlige Identität und Unveränderlichkeit des Denkstils gäbe, würde jede Entdeckung, d.h. das Wahrnehmen von etwas Neuem, unmöglich.» ${ }^{22}$

Ein im Vergleich zum Paradigma offeneres Gebilde stellt der Denkstil nicht nur im historischen Längsschnitt dar, sondern, wie sich weiter zeigen wird, auch in synchroner Richtung. - Fleck spricht zwar vom Denkstil mitunter als «kollektivem Denkzwang» ${ }^{23}$, erkennt jedoch in ihm durchaus die Abstraktion: das Denkkollektiv besteht aus Individuen, und «... das individuelle menschliche Seelenleben enthält inkongruente Elemente ..., die die Reinheit jeder Lehre, jedes Systems trüben ... Ein Individuum gehört eben mehreren Denkstilen an» ${ }^{24}$; und : «jeder Mensch besitzt sogar viele, z. T. einander widersprechende Wirklichkeiten». ${ }^{25}$

Nun haben gerade die Inkohärenzen des individuellen Denkens für die Wissenschaft eine wesentliche, nämlich heuristische Funktion: ohne sie könnte Neues gar nicht wahrgenommen, könnten Entdeckungen nicht gemacht und Denkstile nicht verändert werden. So beschreibt Fleck die kognitive Widersprüchlichkeit der frühneuzeitlichen Anatomen als Voraus- 
setzung für die neuen Entdeckungen: «Wenn wir Arbeiten aus dieser Zeit lesen, scheint es uns, dass die Autoren an einem Schwindel litten, dass es ihnen vor den Augen flimmerte, dass sie die mittelalterliche Welt und den Weg zur neuen Welt abwechselnd sahen.» ${ }^{26}$

Die für die Wissenschaft fruchtbaren Inkohärenzen des individuellen Denkens und Wahrnehmens haben nun ihre Grundlage in Elementen des Denkstils selbst, die über ihn hinausweisen und ihn mit anderen Denkstilen verknüpfen. Es sind die von Fleck so genannten «Präideen», nämlich ursprünglich-archetyphafte, noch polyvalente Begriffe oder Ideen einer Kul$\operatorname{tur}$ (z. B. «Atom», «Masse», «Krankheitserreger» u. a.), deren einzelne Motive immer wieder neu aktualisiert und in divergente Richtungen weiterentwikkelt werden können. Mit zunehmender Differenzierung und Spezialisierung gewinnen die wissenschaftlichen Begriffe an Präzision, verlieren aber an Plastizität und Potenz.

Als ein Beispiel für diesen Prozess wollen wir den auch von Fleck untersuchten Begriff der «Wärme» aufgreifen ${ }^{27}$. Für Antike und Mittelalter ist «Wärme» ein durchaus proteisches Gebilde: ein und dasselbe ist die Wärme des Feuers, die «heisse» Würze der Speisen, die Körper- und Lebenswärme, die «Hitzigkeit» des Temperaments oder auch die mitmenschliche Wärme. Nicht dass sich dabei «eigentliche» und «übertragene» Bedeutung unterscheiden liessen : alle diese Formen von «Wärme» sind letztlich wesensgleich, ja identisch; der Erfahrungsgehalt in diesen verschiedenen Situationen ist (in einer schwer zu bezeichnenden Weise) der gleiche. - Erst im weiteren Verlauf, v. a. in der Neuzeit, differenzieren sich die verschiedenen Bedeutungen und treten zueinander in Widerspruch: die physikalische Wärme hat, als «Teilchenbewegung», nichts mehr gemein mit der «Lebenskraft» (calor innatus) oder der gefühlsmässig erfahrenen Wärme.

Und doch bleibt die Präidee in ihrer Pluripotenz latent erhalten und wirksam; sie wird «zur unbewussten Leitlinie für die Begriffsentwicklung, die sich so gestaltet, dass die Präidee bewiesen werden kann». ${ }^{28}$ - In unserem Beispiel: der Widerspruch zwischen "physikalischer» und «Lebens»wärme (bzw. den ihnen entsprechenden Denkstilen) entfaltete sich aus einer anfänglichen Einheit, die aber als Präidee einer Identität von Feuer und Leben latent fortwirkt. Dies führt schliesslich zum Versuch einer Auflösung des Widerspruchs : nämlich durch die reduktionistische Umdeutung der Lebenswärme in eine Teilchenreaktion, unter dem physikalisch-chemisch neu definierten Begriff der «Verbrennung» (Mayow 1674, Lavoisier 1789). - Ähnliche 
Entwicklungen lassen sich auch an anderen, ursprünglich polyvalenten Begriffen verfolgen, wie denen der «Schwere», der «Masse» oder des «Lichts».

In ihren Konnotationen transportieren Begriffe also immer auch denkstilfremde Gehalte, und gerade dadurch können sie als Verbindungsglieder zwischen gegensätzlichen Denkstilen fungieren. «Jede Epoche hat herrschende Auffassungen, Überreste vergangener und Anlagen zukünftiger.» ${ }^{29}$ In den Mutationen der Denkgeschichte, so können wir Flecks Gedanken weiterführen, treten diese latenten Auffassungen wieder hervor; in einer Regression auf die archaische, polyvalent-plastische Stufe der Begriffsgeschichte können sich neue, zu den bisherigen inkongruente Begriffe konstituieren ${ }^{30}$. So entstehen - oft durch gezielte Umdeutungen - neue Denkstile aus den Elementen alter, und sie bilden ihrerseits das Material für zukünftige.

Halten wir das bisher Ausgeführte gegen Kuhns Paradigmen-Modell, so ergeben sich einige bedeutsame Unterschiede. Zwar sieht auch Fleck die Wissenschaftsgeschichte nicht als stetigen Prozess; doch statt wie die Paradigmen in einem eher disparaten Nacheinander stehen Flecks Denkstile in einem vielfältigen Beziehungsgefüge. Zum einen entwickeln sich widersprechende Sichtweisen aus einer anfänglichen Einheit, die sich in divergente Richtungen auffaltet und so nach und nach die in den Präideen angelegten Möglichkeiten aktualisiert; diese Ideen bleiben aber auch in späteren historischen Phasen latent gegenwärtig und gerade in Denkstilumbrüchen wirksam. Damit wird das Moment der Kontinuität im diskontinuierlichen Ablauf der Wissenschaftsgeschichte begreifbar.

Zum anderen beschränkt Fleck die Koexistenz von Widersprüchen nicht auf die Revolutionen der Wissenschaftsentwicklung; Kuhns reine Monoparadigmatik erschiene aus seiner Sicht als Abstraktion. Vielmehr enthält jeder Denkstil schon für sich genommen inkongruente Elemente und Begriffsgehalte, die als Keime des Widerspruchs fungieren. Darüber hinaus aber kreuzen sich wie in den Begriffen, so auch in den Individuen «...bisweilen widersprechende, manchmal sorgfältig voneinander isolierte Denkstile» ${ }^{31}$ - Inkohärenzen mit wesentlicher heuristischer Bedeutung für den Fortgang der Wissenschaft. Kein Denkstil lässt sich also isoliert für sich, ohne Bezug zu seinen Vorgängern bzw. Kontrahenten begreifen; und anstelle einer seriellen Abfolge einzelner Paradigmen beherrscht die simultane Polyvalenz von Begriffen und Sichtweisen, latent oder offen, letztlich jeden Zeitpunkt der Wissenschaftsgeschichte. 


\section{Fallbeispiel: Vitalismus versus Mechanismus in der Physiologiegeschichte}

Ein historisches Fallbeispiel soll das im letzten Abschnitt Ausgeführte verdeutlichen, aber auch um einen neuen Aspekt erweitern. Weder Kuhn noch Fleck haben nämlich die Möglichkeit einer Interaktion und wechselseitigperiodischen Ablösung zweier Denkstile über einen längeren Zeitraum ins Auge gefasst; eine solche Beziehung aber ergibt sich bei der Verfolgung von Vitalismus und Mechanismus in der Physiologie im Anschluss an die Kreislaufentdeckung William Harveys. Im folgenden soll die historische Dialektik dieser beiden Denkstile kurz skizziert werden ${ }^{32}$.

Harveys Entdeckung des Blutkreislaufs (De Motu Cordis 1628) kann selbst schon als Frucht des Zusammentreffens gegensätzlicher Denkstile in einem Individuum aufgefasst werden: tradierte scholastische Physiologie, aristotelischer Vitalismus, magisch-hermetische Ideen der Renaissancephilosophie und die neuen experimentellen bzw. mechanistischen Tendenzen der Physiologie kreuzten sich in Harveys Denken, ohne dass er sie als so widersprüchlich wahrgenommen hätte, wie wir dies heute täten ${ }^{33}$.

Nicht ohne weiteres kongruent sind auch die beiden wesentlichen Präideen, die in die Kreislaufentdeckung eingeflossen sind : zum einen die antike Idee der himmlischen Kreisbewegung als Urbild einer Eigen-oder Selbstbewegung; zum anderen die aristotelische Auffassung vom Herz als dem Zentralorgan des Körpers (der «Sonne des Mikrokosmos»), die Harvey der galenischen Trias von Leber, Herz und Gehirn entgegenstellt.

Eigenbewegung des Blutes auf seiner Kreisbahn und Antrieb durch das Zentralorgan - aus diesen heterogenen Elementen entsteht Harveys Konzeption des Blutkreislaufs: er bildet sich aus einer primären, embryonalen Eigenbewegung des Blutes, das Harvey als den beseelten Lebensstoff des Organismus auffasst. Diese Eigenbewegung wird dann vom Herz aufgenommen, mechanisch verstärkt und so vom Zentrum auf den ganzen Organismus erweitert. So kommt es zu der zunächst widersprüchlich anmutenden Kombination einer mechanischen Pumpbewegung im arteriellen Kreislauf und einer vitalen Eigenbewegung des zum Herz «strebenden» Blutes im venösen Kreislauf. Als verbindendes Prinzip entwickelt Harvey später den Gedanken einer elementaren Wahrnehmungsfähigkeit (sensus) organischer Gewebe, aufgrund derer das Herz den venösen Bluteinstrom als Reiz empfindet (irritatus) und mit Kontraktion beantwortet ${ }^{34}$.

Zusammengefasst: Die Zurückführung der Autonomie des Organismus auf ein Kreislaufgeschehen in Analogie zur Selbstbewegung der Himmels- 
körper; die Erklärung des Kreislaufs aus der embryonalen Morphogenese und den besonderen Eigenschaften organischer Gewebe bzw. des Blutes; und die Einbettung der mechanischen Kreislaufkomponente in den vitalen Prozess, dem sie ursprünglich entstammt - dies sind die wesentlichen Elemente des Harveyschen Vitalismus.

Dieser vitalistische Neuansatz wurde allerdings in der Folgezeit kaum weitergeführt, so dass er bis in unser Jahrhundert hinein weitgehend vergessen blieb. Der Grund dafür lag in der Dominanz des mechanistischen Denkstils, der, von Descartes inauguriert, in kurzer Zeit die europäische Physiologie eroberte. - Descartes selbst griff als erster Harveys Entdeckung auf, deutete sie jedoch in ganz anderem Sinn. In der Kreisbewegung des Blutes, für Harvey Grundlage der vitalen Autonomie des Organismus, sah Descartes vielmehr die Möglichkeit, erstmals eine mechanische Automatie des Körpers unter Ausschluss vitaler und psychischer Kräfte zu postulieren. Die Präidee des Kreises erschien bei Harvey als die Selbstbewegung der Himmelskörper; bei Descartes hingegen konkretisiert sie sich im Bild des ablaufenden Uhrwerks, mit dem er den Mechanismus der Herzaktion vergleicht ${ }^{35}$. Die Steuerung des Uhrentakts geschieht von aussen, nämlich durch die Herznerven: auf diese Weise deutet Descartes auch die Präidee einer Verbindung von Herz und Seele neu; sie wird zur Leitidee einer neurochemischen Physiologie der Affekte ${ }^{36}$.

In der Folgezeit eroberte der mechanistische Denkstil nach und nach das ihm ursprünglich fremde Territorium des Lebendigen: auch die Physiologen und Mediziner der Zeit waren vom cartesischen Ideal der Einheitswissenschaft fasziniert. Der Kreislauf galt nun allgemein als rein mechanischpassive Blutbewegung in einem Pumpen- und Röhrenapparat; und an die Stelle von Harveys Autonomie des irritablen Herzens trat nun, im Gefolge Descartes', die Theorie der Aussensteuerung des Herzens durch einströmendes Nervenfluidum ${ }^{37}$.

Und doch zeigt die nähere Untersuchung der physiologischen Quellen zwischen 1650 und 1750, dass das cartesische Maschinenparadigma vitalistische Sichtweisen nicht völlig zu verdrängen vermochte, so dass diese als «unterirdische Gegenströmung» weiter wirksam blieben. Sie wird nicht nur im vereinzelten Auftreten vitalistischer Physiologen (wie etwa Francis Glissons in England) sichtbar, sondern auch bei durchaus mechanistisch denkenden Physiologen, die in ihre Erklärungen der Herztätigkeit denkstilfremde Begriffe und inkonsistente Elemente einfügen. So gestehen etwa Franciscus Sylvius oder Johannes Bohn, beide Vertreter der «offiziellen» 
Herztheorie und von Descartes stark beeinflusst, dem Herz doch auch eine Reizwahrnehmung zu, da sie die Besonderheit seiner rhythmisch wiederholten Aktion anders nicht zu erklären vermögen ${ }^{38}$. Ähnlich spricht der Mechanist Giovanni Borelli - unter dem Eindruck der Präidee vom Herz als Sitz der Seele - von einer «seelischen Perzeption» des «irritierenden» Bluteinstroms; zusätzlich zum neuronalen Steuerungsmechanismus der Herzaktion soll damit deren Abhängigkeit von Affekten ihre Erklärung finden ${ }^{39}$.

Das Herz scheint sich also aufgrund seiner rätselhaften Rhythmik und Beziehung zum seelischen Erleben der Reduktion auf den mechanistischen Aspekt in besonderer Weise widersetzt zu haben. Es zeigt sich eine fortdauernde Latenz vitalistischer Ideen, gerade auch dort, wo sie in mechanistischen Zusammenhängen erkennbar werden.

Nur so wird erklärlich, dass es um 1750 zu einem relativ plötzlichen Umschlag kommen konnte. Albrecht von Haller fasste die Ergebnisse seiner Experimente mit Lebendpräparaten unter den Begriffen von «Irritabilität» und «Sensibilität» zusammen und nahm damit - ohne sich dessen noch bewusst zu sein - über Francis Glisson als Mittler die Harveysche Konzeption einer elementaren Sensibilität organischer Gewebe wieder auf; ebenso im übrigen Harveys Erklärung der Herztätigkeit als Wechselspiel von Bluteinstrom und Herzkontraktion ${ }^{40}$.

Nun war Haller eigentlich Mechanist - doch der latent vitalistische Gehalt seiner Begriffe trat in plötzlichem, zeitbedingtem Bedeutungswandel hervor. Ja, im Rückblick wird erkennbar, dass bereits in Hallers Sichtweise Widersprüchlichkeiten und Inkohärenzen bestanden, die ihm seine Entdekkungen überhaupt erst ermöglichten. - Gegen seine Intention wurden seine Ergebnisse allgemein als Beweis für spezifisch vitale Kräfte aufgefasst, die den bisherigen Dualismus von Seele und Körpermaschine in Frage stellten; in kurzer Zeit stieg nun der Vitalismus zur dominierenden Sichtweise auf.

Doch bedeutete dies keine Wiederkehr des Gleichen, keinen einfachen Paradigmenaustausch: der vitalistische Denkstil zeigte sich in vieler Hinsicht beeinflusst und modifiziert durch die abgelaufene mechanistische Phase. Dies wird etwa an einer veränderten Auffassung von «Irritabilität» deutlich: an die Stelle unbewusst-organischer «Wahrnehmung» trat zunehmend der Automatismus von Reiz und Reaktion, also ein quasimechanischer Ablauf. - Den nochmals radikalisierten Vitalismus der romantischen Physiologie (etwa 1790-1830) konnte daher Hallers Erklärung der Herzaktion nicht mehr befriedigen; statt dessen griff man auf die galenische «facultas pulsifica» zurück und postulierte eine gänzlich autonome Eigenbe- 
wegung des Herzens. «Irritabilität» sollte nun «Selbst-Erregung» bedeuten - eine erneute, folgenreiche Umdeutung des Begriffs ${ }^{41}$.

Der um 1830/40 in der Physiologie wieder zum Durchbruch gelangende Denkstil knüpfte trotz vieler Modifizierungen in seinen Grundlagen an die mechanistische Tradition an - auch wenn seinen Exponenten die «unterirdische» Verbindung zu Descartes (analog zur Latenz des Vitalismus zwischen 1650 und 1750) nicht mehr bewusst war. Sie wird nur zu deutlich etwa an folgender Äusserung François Magendies :

«Und ist nicht jene hydraulische Maschine, die dazu bestimmt ist, das Blut in unserem Gewebe zirkulieren zu lassen, ein Wunderwerk in der Mechanik? ... selbst ohne vitale Gesetze (müsste) die Zirkulation am Leichnam ... bestehen können, wenn man im Stande wäre, das ganze System von Pumpen und Röhren ... in Bewegung zu setzen.» ${ }^{42}$

Es wäre wohl zu erwarten, dass dieses Paradigma auch die mechanistische Theorie einer zentralnervösen Steuerung des Herzens wiederaufnähme - sie war während der vitalistischen Phase latent geblieben und um 1800 vereinzelt wieder aufgetaucht ${ }^{43}$. Tatsächlich aber kam es zu einer erneuten Interaktion der beiden gegensätzlichen Sichtweisen : aus den Präideen einer «Selbsterregung» des Herzens einerseits, einer zentralnervösen Steuerung andererseits bildete sich die neue Legierung eines autonomen nervösen Steuerungszentrums im Herzen, welches von Volkmann 1850 postuliert und von Stannius 1852 im Sinusknoten schliesslich auch lokalisiert wurde ${ }^{44}$.

Damit sind wir in unserem historischen Überblick bei dem heute noch herrschenden Paradigma angekommen, dessen Selbstverständnis abschliessend durch ein Zitat des bedeutenden Pathologen Bernhard Naunyn (18391925) verdeutlicht werden soll:

\footnotetext{
«Unsere Heilkunde ist das, was sie geworden ist, geworden seitdem sie sich der Führung der Naturwissenschaften anvertrauen konnte ... Das konnte nicht früher geschehen als in der ersten Hälfte des vergangenen Jahrhunderts ... Von da beginnt die moderne Ära der Medizin, seitdem vollzieht sich ihre Entwicklung in ungestörter Kontinuität, ... ununterbrochen, sicher und erstaunlich gross war bisher der Fortschritt und er wird es auch ferner bleiben, solange wir unserer Fahne, der Fahne der Naturwissenschaften, treu bleiben.» ${ }^{45}$
}

Hier finden sich noch einmal die wesentlichen Elemente des mit der neuzeitlichen Wissenschaft verknüpften Anspruchs: Kontinuität, stetiger Fortschritt, Geschlossenheit und das Verbot der Häresie. - Dessenungeachtet hatte seither nicht nur die Medizin insgesamt, sondern auch die Physiologie insbesondere durchaus mit abweichenden Standpunkten zu kämpfen. So setzte mit dem Neovitalismus zu Beginn des Jahrhunderts erneut die Kritik an der mechanistischen Kreislauf-Konzeption ein; und auch gegenwärtig 
wird ihr wieder der Primat einer peripheren und nicht vom Herz angetriebenen Blutströmung entgegengestellt - der alten vitalistischen «Selbstbewegung» des beseelten Blutes entsprechend. Für den Historiker wäre es vermessen zu glauben, dass solche Aussenseiterstandpunkte für alle Zukunft ihre Rolle ausgespielt hätten ${ }^{46}$.

\section{Ergebnisse und Folgerungen}

Als Zusammenfassung unserer Fallstudie lassen sich folgende Ergebnisse formulieren:

Die Entwicklung der Physiologie seit der Neuzeit folgt nicht einem kontinuierlichen Verlauf. Selbst auf dem Gebiet der Herz- und Kreislaufphysiologie wechseln auf der unumstrittenen Basis von Harveys Entdeckung doch unterschiedliche Erklärungsansätze einander ab. Dennoch entspricht das Bild auch nicht ganz dem Kuhnschen Modell einer disparaten Paradigmen-Abfolge.

Zum einen finden sich nämlich denkstilübergreifende Elemente (Präideen wie etwa Kreisbewegung; Herz als Wärme-, Herz als Gefühlsorgan; Irritabilität, Sensibilität), die in den Paradigmen gegensätzliche Bedeutung gewinnen. Durch Reaktualisierung ihrer ursprünglichen «Unschärfe» entsteht in Zeiten des Denkstilwandels das «kreative Chaos», in dem sich v.a. durch Umdeutungen neue Sichtweisen bilden können.

Zum anderen zeigt sich das Phänomen einer periodischen Wiederkehr und Interaktion zweier Denkstile. Keiner vermochte den anderen auf die Dauer zu verdrängen; im Gegenteil sah jeder sich genötigt, im Laufe seiner Entwicklung auch Gehalte des Konkurrenten mitaufzunehmen. - Eine solche «diachrone Konkurrenz» liesse sich auch in anderen Wissenschaftsbereichen aufweisen. Zu erinnern wäre an den Widerstreit von Nahkausalität und Fernwirkungsprinzip in der Physik ${ }^{47}$, von qualitativ orientierter und atomistischquantifizierender Chemie, oder an den Wechsel von Krankheitsauffassungen in der Medizingeschichte: Krankheit als Kampf gegen äussere Angreifer (Dämonen, Miasmen, Viren ...) oder als primäre Störung innerorganismischen Gleichgewichts (der Säfte, der psychosomatischen Ganzheit o.ä.). Offenbar wird die Wissenschaftsentwicklung in solchen Fällen gespeist von einer Polarität, deren beide Momente fortwährend aufeinander bezogen bleiben. Darauf deutet noch ein weiteres Ergebnis unserer Fallstudie hin, 
nämlich die Latenz des scheinbar ausgeschlossenen Kontrahenten in Form von Inkohärenzen des herrschenden Denkstils oder von offenen Häresien.

Diese Ergebnisse könnten dazu geeignet sein, die Kuhnsche Konzeption der Wissenschaftsgeschichte als nur idealtypisch gültig zu erweisen: Nach unserer Auffassung stellt die monoparadigmatische Entwicklung eher einen Grenzfall dar, dem die europäische Wissenschaft seit der Neuzeit zwar zustrebt, den sie jedoch nie wirklich zu erreichen vermag. Verdeckt durch die Dominanz eines Denkstils, bestehen die Widersprüche latent weiter fort; und periodisch kehren bestimmte Grundtypen von Denkstilen wieder. Ja, ohne diese Latenz wäre gar nicht erklärbar, wie es in den Krisen der Wissenschaftsgeschichte zum plötzlichen Auftreten konkurrierender Sichtweisen kommen kann.

Wenn diese Annahmen in allgemeinerer Form zutreffend sind, verläuft die Wissenschaftsgeschichte unter der Oberfläche wesentlich komplexer, als es den Anschein hat. Dies könnte geeignet sein, Kuhns monoparadigmatische Konzeption in ihrer Normativität für die Wissenschaftsentwicklung in Frage zu stellen: die ohnehin bestehende diachrone Konkurrenz lässt auch einen pluralistischen Wissenschaftsbegriff möglich erscheinen, der eine Koexistenz von älteren, bewährten und jüngeren, innovativen Erklärungsansätzen zuliesse. Aussereuropäische Kulturen wie etwa die chinesische, scheinen dieser Möglichkeit tatsächlich in einem hohen Masse Raum gegeben zu haben ${ }^{48}$. Gründe für die Aufrechterhaltung des westlichen Erklärungsmonismus dürften demgegenüber in theoretischen wie praktischen Erwägungen bestehen, die abschliessend kurz erörtert werden sollen.

1. Theoretisch liegt dem Erklärungsmonismus v.a. die ontologische Überzeugung von der Einheit einer absoluten Wirklichkeit zugrunde, die letztlich auch nur durch eine Sichtweise fortschreitend erfasst oder wenigstens durch ein Erklärungsmodell am adäquatesten abgebildet werden könne. - Allerdings kann die Einsicht in die Diskontinuität der Wissenschaftsgeschichte und in die historische Relativität der Denkstile die Konsequenz eines erkenntnistheoretischen Skeptizismus nur vermeiden, wenn die Wirklichkeit selbst als heterogen, komplex und polyvalent gedacht wird - so dass durch die verschiedenen Sichtweisen eben nur jeweils bestimmte Aspekte dieser Wirklichkeit erfasst werden.

Eine solche Wirklichkeitsauffassung wird auch durch die zuvor geschilderte historische Dialektik konkurrierender Perspektiven gestützt: gerade durch den Ausschluss anderer Erklärungsweisen gerät nämlich das jeweils herrschende Paradigma mehr und mehr in den Zwang, auch Phänomene 
ausserhalb seines ursprünglichen Wirklichkeitsausschnitts deuten und erklären zu müssen (so etwa der Mechanismus zwischen 1650 und 1750). Diese aber sind seiner besonderen Perspektive inadäquat und können nur noch verzerrt oder reduktionistisch erfasst werden. Hierin dürfte ein wichtiger Grund für die von Kuhn beschriebenen «Anomalien» und die schliessliche Krise eines Paradigmas liegen. - Umgekehrt bedeutet die Renaissance einer verdrängten Sichtweise, dass sie tatsächlich einen Aspekt der Wirklichkeit zu erfassen vermochte, der sich in der Krise nun wieder als eigenständiger geltend macht. - Der besondere, periodisch-krisenhafte Verlauf der abendländischen Wissenschaftsgeschichte hätte seine Ursache somit gerade in deren monistischer Tendenz!

2. Unter pragmatischen Gesichtspunkten kann sich der Erklärungsmonismus durch Verweis auf die Vorteile der Geschlossenheit legitimieren : höhere Effektivität konsensorientierter Forschung; Konzentration statt Zersplitterung gesellschaftlicher Ressourcen; Eindeutigkeit praktischer Konsequenzen und Handlungsanweisungen im Gefolge wissenschaftlicher Forschung.Diese Vorteile dürften in der Tat einen entscheidenden Anteil am Erfolg der Naturwissenschaften tragen. Allerdings sind sie mit gewichtigen Nachteilen verbunden: Verdrängung bewährter Erkenntnissysteme und Handlungskonzepte, Einseitigkeit des Blickfeldes und Ausblendung von Wirklichkeitsbereichen, insbesondere aus der Planung und Folgenabschätzung wissenschaftlich-technischen Handelns. Der grösseren Effizienz des wissenschaftlichen Monismus steht also möglicherweise die höhere «Wirklichkeitsadäquatheit» eines wissenschaftlichen Pluralismus gegenüber.

Die Alternativen im Umgang mit der möglichen Vielfalt von Sichtweisen sind jeweils Ausdruck langfristiger Grundhaltungen einer Kultur und daher durch Argumentationen kaum beeinflussbar. Es scheint aber, dass sich auch in unserer Kultur nach den besonderen Entwicklungen des 20. Jahrhunderts eine «postmoderne» Wendung zu einer «Polyperspektivik» im Bereich der Wissenschaften abzeichnet. Eine aspektdifferenzierende Wirklichkeitsauffassung könnte dazu beitragen, unterschiedliche Perspektiven weder als sich gegenseitig bedrohend und aufhebend, noch als sich bis zur Beliebigkeit relativierend zu begreifen. Die einander zunächst inkommensurablen Sichtweisen könnten komplementäre Beiträge zu einem reichhaltigeren Wirklichkeitsbild liefern, ohne den Anspruch auf Wahrheit, nämlich adäquate Erkenntnis der Wirklichkeit je unter einem besonderen Aspekt, aufzugeben. Fruchtbarer Widerstreit träte an die Stelle möglichst rasch zu überwindenden Widerspruchs. Den Begriffen des linear-kumulativen oder des nur inner- 
paradigmatischen Fortschritts liesse sich dann, als ein Drittes, das Bild einer komplementären Evolution gegenüberstellen, in der die historische Entfaltung gegensätzlicher Sichtweisen die Einheit der Wirklichkeit um so reicher hervortreten lässt. 


\section{Anmerkungen}

1 Vgl. etwa R. Specht, Innovation und Folgelast. Stuttgart 1972, oder G. Böhme, W. van den Daele, W. Krohn, Alternativen in der Wissenschaft. In : Zt.f. Soziologie 1/1972, S. 302-316: «Die Wissenschaft entwickelt sich über eine Kette von Verzweigungsstellen, ... an denen sich einheitliche Forschungsstränge spalten können zu disparaten Theorielinien, an denen aber auch liegengebliebene Ansätze - «tote Äste der Wissenschaftsgeschichte〉 - wieder aufgenommen werden können ...» (S. 304).

2 R. Descartes, Discours de la Méthode (1637), dt. Übs. Hamburg 1960, S. 51 (VI, 2).

3 F. Bacon, Novum Organon (1620), in: The Works of Francis Bacon, Bd. I, Stuttgart 1963; dt. Neues Organon der Wissenschaften, Darmstadt 1981, S. 74 (I, 94).

4 Ebd. S. 58 (I, 78).

5 Ebd. S.56 (I, 76 f.).

6 Vgl. R.Specht, René Descartes. Hamburg 1966, S. 17 ff.

7 R. Descartes, Principia Philosophiae (1644), dt. Übs. Hamburg 1955, S. XLV.

8 Ebd.

9 Vgl. M. Ritter, Fortschritt. In: Ders. (Hg.), Historisches Wörterbuch der Philosophie. Darmstadt 1972, Bd.2, S.1032-1059; S. 1048.

10 P. Feyerabend, Wider den Methodenzwang. Skizze einer anarchistischen Erkenntnistheorie. Frankfurt 1976.

11 Vgl. zum Folgenden T. S. Kuhn, Die Struktur wissenschaftlicher Revolutionen. Frankfurt (2) 1976, sowie ders., Die Entstehung des Neuen. Frankfurt 1977.

12 Kuhn (1976) S. 35, (1977) S. 315 f.

13 Ebd. S.18.

14 Kuhn (1977) S.316.

15 Kuhn (1976) S. 182.

16 Ebd. S. $168 \mathrm{f}$.

17 Ebd. S. 182.

18 Ebd. S.178.

19 «Einige Leser haben festgestellt, dass ich wiederholt zwischen deskriptiver und normativer Darstellung wechsle ... Die obigen Darlegungen stellen ... eine Theorie über die Natur der Wissenschaft dar, und wie andere Wissenschaftstheorien enthält sie Konsequenzen für die Verhaltensweisen von Wissenschaftlern, wenn sie erfolgreich sein wollen. Obgleich sie nicht richtiger zu sein braucht als andere Theorien, liefert sie doch die legitime Basis für die wiederholte Verwendung von Ausdrücken wie «sollte sein`.» Ebd. S.218f.

20 Vgl. zum Folgenden L. Fleck, Entstehung und Entwicklung einer wissenschaftlichen Tatsache (1935). Frankfurt 1980; ders., Erfahrung und Tatsache. Frankfurt 1983.

21 Fleck (1980) S.38, S. 121.

22 Fleck (1983) S.68.

23 Fleck (1980) S. 124, S. 131 f.; (1983) S. 157.

24 Fleck (1980) S. $60 \mathrm{f}$.

25 Fleck (1983) S. 48.

26 Ebd. S. 78; vgl. auch Fleck (1980) S.124f.

27 Fleck (1983) S. $98 \mathrm{ff}$. 
sound and relevant with its apparent opposite must have been possible in the same mind
which yet somehow retained its integrity and power." - W. Pagel, New Light on William
Harvey. Basel 1976, S. 1 .

34 W. Harvey, De Generatione Animalium (1651), Kap. 51; in: The Works of William Harvey, hg. R. Willis, London 1965, S. 375 ff.

35 Discours de la Méthode, S.41 (V, 6).

36 Vgl. R. Descartes, Les Passions de l'Ame (1649).

37 So bei Henricus Regius, Franciscus Sylvius, Cornelis Bontekoe, Nathaniel Highmore, Thomas Willis, Richard Lower, John Mayow, Johannes Bohn, Friedrich Hoffmann, Herman Boerhaave u. a. - vgl. Fuchs (1992), S. 143-186.

38 Franciscus Sylvius, Disputationes Medicae (1659ff.), III, 15; Johannes Bohn, Circulus anatomico-physiologicus (1686), S.449f.

39 G. Borelli, De Motu Animalium (1680/81), Leyden 1685, Bd. II, S. 116 f.

40 A.v. Haller, Primae lineae Physiologiae. Göttingen 1751, p. 67.

41 Vgl. v. a.P. F. v. Walther, Physiologie des Menschen, Landshut 1807/08, Bd. II, S. $31 \mathrm{ff}$.

42 F. Magendie, Vorlesungen über die physikalischen Erscheinungen des Lebens. Köln 1837, S. 12 .

43 So etwa bei C. Legallois, Expériences sur le Principe de la Vie. Paris 1812.

44 H.F. Stannius, Zwei Reihen physiologischer Versuche. In: Müllers Archiv, Berlin 1852, S. 85-100.

45 B. Naunyn, Moderne Kliniken und Krankenhäuser. Rede 1902. In: Ges. Abh. Bd. II, S.1311; zit. nach K.E. Rothschuh, Konzepte der Medizin in Vergangenheit und Gegenwart. Stuttgart 1978, S. 429 (Hvhb. v. Vf., T. F.).

46 Vgl. u.a. E. Pestel, G. Liebau (Hgg.), Phänomen der pulsierenden Strömung im Blutkreislauf aus technologischer, physiologischer und klinischer Sicht. Mannheim 1970; F. Husemann, O. Wolff, Das Bild des Menschen als Grundlage der Heilkunst. Stuttgart 1986, Bd.III, S. 95 ff., 144 ff.; L. Manteuffel-Szoege, Über die Bewegung des Blutes. Stuttgart 1977.

47 Vgl. A. Diemer (Hg.), Der Methodenpluralismus in den Wissenschaften. Düsseldorf 1970.

48 Nach P. U. Unschuld ist besonders die Entwicklung der chinesischen Heilkunde durch eine «wachsende synchrone Vielfalt» unterschiedlicher medizinischer Ideensysteme charakterisiert (Medizin in China. Eine Ideengeschichte. München 1980, S.12). Das traditionelle chinesische Denken vollzieht sich nach Unschuld in einer «polylinearen Logik, die die 
endgültige Annahme mehrerer, auch einander ausschliessender Erklärungsmodelle erlaubt» (Gedanken zur kognitiven Ästhetik Europas und Ostasiens. In: Jahrbuch 1988 der Akademie der Wissenschaften zu Berlin. Berlin 1989, S.352-366).

Dr. Thomas Fuchs

Psychiatrische Klinik und Poliklinik der

Technischen Universität

Ismaninger Strasse 22

D-81675 München 